\title{
Advancements on the simulation of the micro injection moulding process
}

\author{
Marhöfer, David Maximilian; Tosello, Guido; Hansen, Hans Nørgaard; Islam, Aminul
}

Published in:

Proceedings of the 10th International Conference on Multi-Material Micro Manufacture

Publication date:

2013

Link back to DTU Orbit

Citation (APA):

Marhöfer, D. M., Tosello, G., Hansen, H. N., \& Islam, A. (2013). Advancements on the simulation of the micro injection moulding process. In Proceedings of the 10th International Conference on Multi-Material Micro Manufacture 4M Association.

\section{General rights}

Copyright and moral rights for the publications made accessible in the public portal are retained by the authors and/or other copyright owners and it is a condition of accessing publications that users recognise and abide by the legal requirements associated with these rights.

- Users may download and print one copy of any publication from the public portal for the purpose of private study or research.

- You may not further distribute the material or use it for any profit-making activity or commercial gain

- You may freely distribute the URL identifying the publication in the public portal

If you believe that this document breaches copyright please contact us providing details, and we will remove access to the work immediately and investigate your claim 


\title{
Advancements on the simulation of the micro injection molding process
}

\author{
D.M. Marhöfer, G. Tosello, H.N. Hansen, A. Islam \\ Technical University of Denmark (DTU), Department of Mechanical Engineering, \\ Produktionstorvet, Building 427A, 2800 Kgs. Lyngby, Denmark
}

\begin{abstract}
Process simulations are applied in micro injection molding with the same purpose as in conventional injection molding: aiming at optimization and support of the design of mold, inserts, plastic products, and the process itself. Available software packages are however not well suited for micro injection molding, because they are developed for macro plastic parts and they are therefore limited in the capability of modeling the polymer flow in micro cavities properly. However, new opportunities for improved accuracy have opened up due to current developments of the simulation technology. Hence, new strategies and aspects for comprehensive simulation models which provide more precise results for micro injection molding are discussed. Modeling and meshing recommendations are presented, leading to a multi-scale mesh of all relevant units in the injection molding process. The implementation of the process boundary conditions is described, being followed by results illustrating their importance on the simulation output. Finally, the influence of the cooling simulation settings is analyzed.
\end{abstract}

Keywords: micro injection molding, simulation, meshing, cooling.

\section{Introduction}

Micro injection molding ( $\mu \mathrm{IM})$ is an efficient replication technology for the mass production of micro plastic parts. Because of its increasing importance and application in different fields of micro technology, simulating the $\mu \mathrm{IM}$ process becomes more and more interesting and relevant. Similarly to conventional injection molding, simulations of $\mu \mathrm{IM}$ aim at reducing micro product time-to-market and optimize resources by avoiding or reducing re-engineering as well as development time [1]. The ability of optimizing mold design and process settings before the production of the mold and the plastic parts is of great advantage.

Nevertheless, $\mu \mathrm{IM}$ process simulations still yield improper quantitative results. This is due to the development of available simulation software packages for macroscopic plastic products. Relevant microscopic effects are not or only inadequately implemented. Up to now, just qualitative predictions about the behavior of polymer and process can be made for micro products, e.g. with high aspect ratios surface structures.

However, the numerical results can be significantly improved by implementing the whole injection molding system and applying the right strategy while building up the model [2].

Usually, the simulation either completely lacks the modeling of feed system (e.g. sprue, runner, gate, additional structures for ejection), mold structures (e.g. block, cooling channels, cartridge heaters), and machine parts (e.g. nozzle, barrel) or their implementation is done insufficiently.

The simulation is very important in mold, part, and process design, so that the development of comprehensive simulation procedures and advanced models suitable for $\mu \mathrm{IM}$ is necessary.

\section{2. $\mu \mathrm{IM}$ process: simulation and experiments}

The commercially available software Autodesk Simulation Moldflow Insight $2013^{\circledR}$ (ASMI) is used for the present work. It provides the possibility to carry out simulation of filling, packing, warpage, and cooling of the injection molding process of macro parts.

Nonetheless, it is possible to implement a simulation model that is able to predict the nature of $\mu \mathrm{IM}$ more correctly. This work outlines in the following sections how to take every system-relevant part into consideration and how to model them by exploiting the available ASMI tool box. Special focus is on meshing, process conditions, and cooling.

The study case was a micro channel test structure with channels and walls of various widths and depths which represent different microfluidic structures (see Fig. 1). Experiments were conducted on a standard injection molding machine (Engel ES 80/25 HL-Victory, $18 \mathrm{~mm}$ screw diameter) using the acrylonitrile butadiene styrene (ABS) Novodur P2H-AT polymer grade. Melt and mold temperature of the simulations were set to $220^{\circ} \mathrm{C}$ and $80^{\circ} \mathrm{C}$, in accordance with the

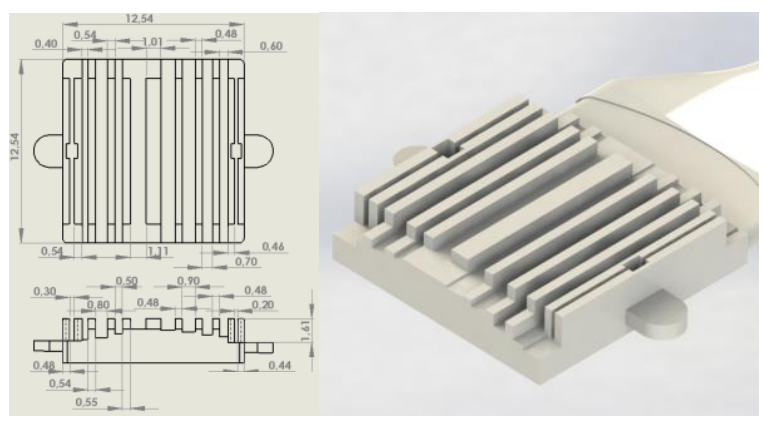

Fig. 1. Technical drawing of molded plastic part (left) and 3D view of part with surface micro structures (right). 
mean values measured by the machine $\left(220.0^{\circ} \mathrm{C} \pm 0.1^{\circ} \mathrm{C}\right.$ and $82.0^{\circ} \mathrm{C} \pm 1.9^{\circ} \mathrm{C}$, respectively).

\section{Modeling of injection molding system}

If macroscopic plastic parts are investigated in simulations, the part itself is normally modeled in 3D by a so-called tetrahedral representation. Contrariwise, the feed system is only represented in a simplified way and modeled in 1D by so-called beam elements or sometimes even completely neglected.

This is due to the little effect of the feed system on the injection. It has only small volume compared to the part, so that assumptions are introduced and faster computation is achieved.

With respect to micro plastic parts, the feed system is of much larger volume than the actual part. It has hence also large influence on the simulation results (e.g. filling time). As a consequence, 3D modeling of the entire part including the feed system becomes necessary to obtain the required accuracy [2].

\section{Meshing guidelines and multi-scale mesh}

The mesh is the first key for successful simulations, since a poor mesh leads to low accuracy results. An optimized mesh density mesh is crucial for and optimal trade-off between computation time and simulation precision.

The first mesh determines the reproducibility of the original $C A D$ shape of the investigated part. The geometry given by the first meshing is maintained at all subsequent operations and also at any remeshing steps. Regarding micro parts, a fine mesh is consequently necessary from the very beginning to reach sufficient contour accuracy. Very important parameters to take into account are:

- the global edge length for general accuracy,

- the chord height for the reproducibility of curved surfaces,

- the merge tolerance for micro features, and

- the bias ratio for thin sections.

The bias ratio is the ratio of element size in the center and at the part surface along the part thickness. It gives rise to the possibility of enhancing the mesh density at the part surface, the more crucial area of the polymer flow, where it interacts with the mold surface. The result of bias at a mesh is shown in Fig. 2. The number of elements and computation time remains constant though. The bias ratio is of great importance for micro parts, because the surface-to-volume ratio is commonly larger than for macro parts [3].

Meshing an entire $\mu \mathrm{IM}$ system including part, feed system, mold structures, and machine parts by a very fine mesh is barely feasible. This yields too many elements and thus unmanageable computation time. The idea of mesh optimization by a multi-scale mesh is inevitable, because multiple parts of very different size are involved in the system. Not every part can be reasonably meshed by one and the same mesh size.

A multi-scale mesh combines therefore a fine mesh at micro features, curved surfaces, and short edges and a coarse mesh at feed system and less

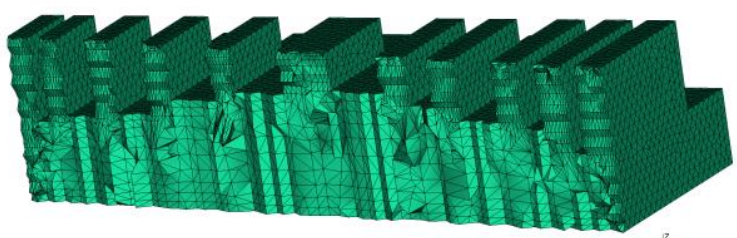

(2.

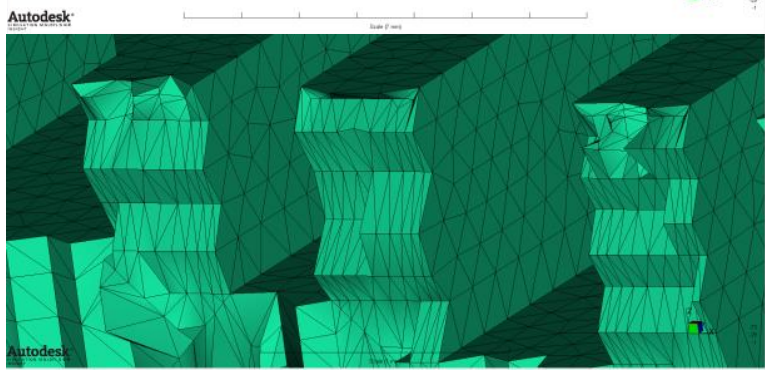

Fig. 2. Effect of bias: different element size across the thickness of part (top); closer view on $\mu$-features with fine mesh (bottom).

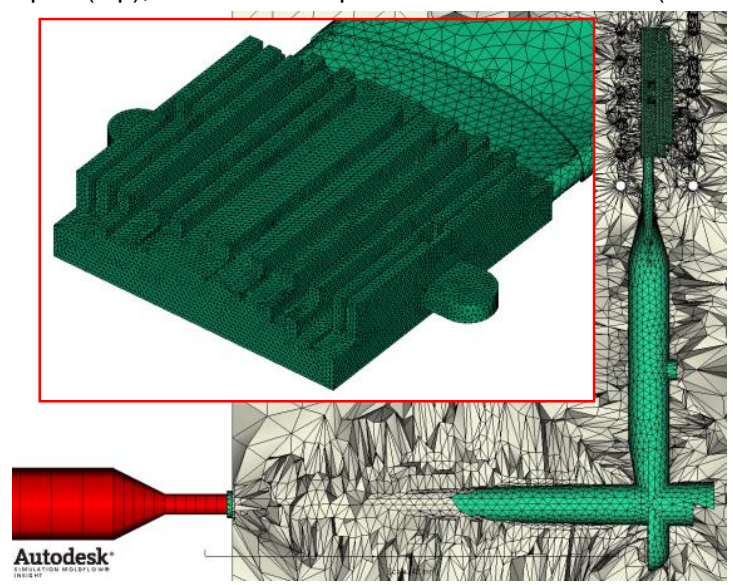

Fig. 3. Multi-scale mesh, overview of nozzle, sprue, mold (coarse mesh) and part (fine mesh).

relevant areas (see Fig. 3).

To do so, some obstacles have to be overcome, as different approaches towards a multi-scale mesh are possible. The starting mesh size can be chosen differently, being followed by one or more remeshing steps. However, the remeshing performance of the software is limited and sometimes there is even no remeshing effect. It appears that the mesh density tool available in the ASMI software leads to the best results. It enables to set the mesh density of different areas to different values before meshing.

In order to achieve the most precise results in $\mu \mathrm{IM}$ simulations, a comprehensive model including all elements involved in the process must be set up. The part should even be replaced by the actual cavity dimensions, if available. The part (or cavity) and the feed system are modeled by 3D representation. The mold is also modeled in 3D as solid block with its actual dimensions and material, including also the real cooling channel layout and possible cartridge heaters. Cooling channels and heaters are modeled by 1D beam elements. Furthermore, the simulation should contain a 1D representation of the actual nozzle geometry of the used injection molding machine and the used stroke volume in the barrel. Fig. 4 shows an 


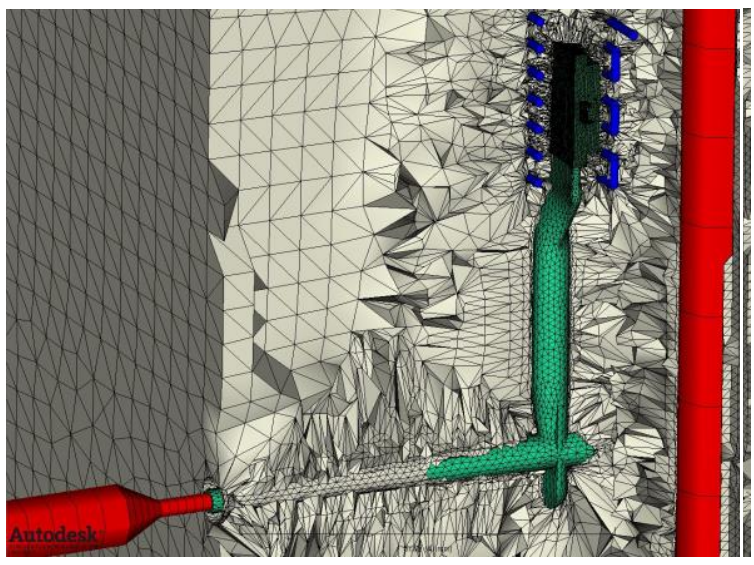

Fig. 2. Comprehensive full 3D simulation model with part and feed system (green/center), cooling (blue/top), and heater (right/red) embedded in mold with additional machine geometry (left/red).

example of how all the parts can be implemented.

\section{Implementation of process boundary conditions}

\subsection{Injection molding machine}

When an injection molding machine is selected from the database of ASMI, different parameters (e.g. stroke length, machine control, hydraulic pressure, screw diameter) can be set. Especially in case of $\mu \mathrm{IM}$, this data and the software's internal considerations are not sufficient to describe the machine properly and to give precise results, since major influences such as the delay due to the actual machine acceleration and the resulting actual polymer flow rate are not taken into account [2].

It is hence recommended to implement manually the speed profile of the injection molding machine based on experimental data to reflect its characteristics as realistic and precisely as possible (see Fig. 5).

As suggested, it is advantageous with regards to the prediction of injection pressure to model nozzle and barrel with their actual geometry, since it accounts better for the pressure drop along the nozzle and barrel than the software's internal assumptions. Precise injection pressure simulation is important, because it is directly linked to the part quality and the crucial process parameter [2]. Besides, this method also simulates better the compressibility of polymer



Fig. 5. Average experimental injection profile (speed repeatability $\pm 0.5 \mathrm{~mm} / \mathrm{s}$ ). between the screw and the nozzle as well as in the melt cushion during both injection and packing.

Yet, this procedure of modeling must be still seen critical and applied carefully, because in ASMI the barrel volume stays constant, whereas in reality the barrel volume decreases during injection.

\subsection{Venting}

ASMI enables to simulate the influence of venting on the filling of the examined part. Full venting of the mold is assumed as default setting. If the mold contains venting structures, it makes sense to include the venting analysis with venting channels based on the real dimensions. If no venting structure exists in the mold, the air in the cavity escapes through the gap between the two closed halves of the mold, when the plastic is injected. This gap and hence the pressure drop depends inter alia on the clamping force and the surface roughness of the mold plates. The flow resistance of the gap can have tremendous influence on the injection pressure, as the machine has to exceed the counter pressure built up by the compressed air in the cavity.

\section{Results and discussion}

It can be observed in the simulation results that the machine geometry and the venting analysis both improve the results, as it is shown in Fig. 6. The simulations clearly yield lower values for all three investigated measures. The part weight is most stable and already the default simulation setup reaches $96 \%$ of the actual value.

The implementation of the machine geometry improves the injection pressure and the injection time by $22 \%$ and $15 \%$, respectively. Although no venting structures are existent in the mold, implementing a venting analysis was found to be necessary, as full venting cannot be assumed. Depending on the height of the venting channel ( $5 \mu \mathrm{m}$ and $2 \mu \mathrm{m})$ modeling the parting gap of the mold, the venting analysis is capable of improving the output again by up to $30 \%$ and $9 \%$. A very small venting channel (denoted as (S) ) illustrates the impact and importance of the venting analysis on the injection pressure.

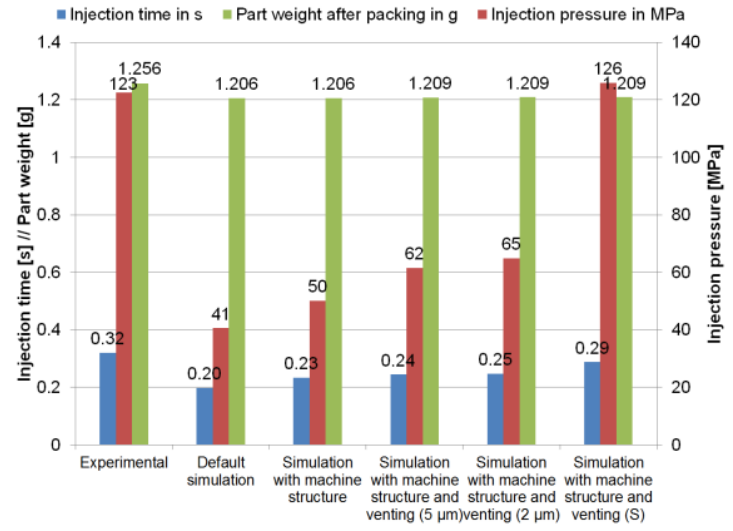

Fig. 6. Injection time, part weight, and injection pressure of experiments and different simulation setups. 


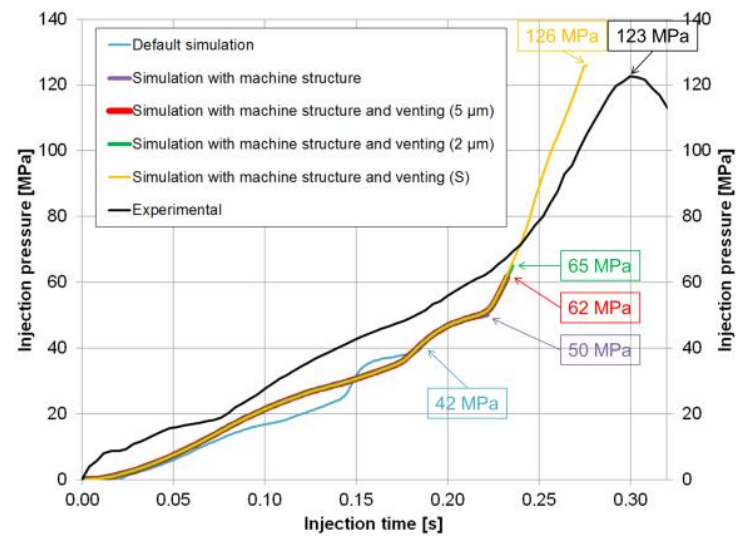

Fig. 3. Injection pressure over time for experiments and different simulation setups during filling.

\section{7.. Cooling and mold block}

In injection molding simulations, the modeling of cooling channels is limited to basic 1D beam element representation. This is sufficient for macro parts and traditional molds with a simple network of straight cooling channels of elementary cross-section. The cooling circuit can be enhanced by bubblers or baffles [4]. Yet, the layout possibilities are still limited.

When applying the novel approach of conformal cooling, the cooling channels follow the contour of the plastic part. This results in very homogenous cooling which can reduce stress and warpage of the part and the cycle time [5]. Conformal cooling is not yet implemented in $\mu \mathrm{IM}$ though.

However, the exploitation of conformal cooling and the exact modeling of cooling channels are especially in $\mu \mathrm{IM}$ of great interest. First, tight tolerances claim good warpage control. Moreover, it often relies on the variotherm process control where cooling and heating phases alternate in accordance to the injection cycle in order to improve part quality $[4,6]$.

Selective laser sintering (SLS) enables the layer by layer fabrication of high quality steel molds with integrated cooling channels of complex shape and at any desired location [4]. Due to improved resolution and decreasing costs, the process becomes suitable for the production of molds with conformal cooling structures, also for micro plastic parts.

An application of full 3D representation of cooling circuits has been described for conventional macro injection moulding [5]. However, the validity of different conformal cooling circuits design suitable for micro injection molding is yet to be proved. This will finally make the simulation of elaborate cooling layouts possible and make conformal cooling employed in $\mu \mathrm{IM}$ industrial application.

\section{Conclusions}

The meshing and modeling of all relevant units (part, mold, machine) of the injection molding process was implemented. It was based on the ability to create a multi-scale mesh with a mesh size in the millimeter range down to micrometer range. The size depends on the importance of the structure and the unit that is meshed.

The scope and method of implementation of the simulation model can have large impact on the simulation results. Regarding $\mu \mathrm{IM}$, all relevant units of the injection molding system must be integrated in the model. Otherwise, the software will yield imprecise results due to its purpose for macroscopic plastic parts. By incorporation of the actual process boundary conditions, machine geometry, and machine behavior, the results can be improved significantly, as well.

Commercially available software provides only simple 1D modeling of cooling channels. The future 3D modeling of cooling channels will enhance the simulation model and open up new possibilities in conformal cooling.

The presented new simulation model and software functionalities are also accompanied by new questions, e.g. about the necessity of modeling molds by CAD files or the adequateness of simpler representations. The necessity for new parameters have also arisen and their influence on the simulation needs to be addressed in the future, e.g. internal roughness of cooling channels, mold material and its HTC (heat transfer coefficient), size of venting channels, as well as the influence of the gap between two mold plates without actual venting structures.

\section{Acknowledgements}

This paper reports work undertaken in the framework of the project "Hi-MICRO" (High Precision Micro Production Technologies, http://www.himicro.eu/), Task 1.2: Micro Injection-Moulding Oriented Product Design. Hi-MICRO is a collaborative project supported by the European Commission in the 7th Framework Programme (Grant agreement no: 314055). Contribution from the consortium which provides useful input and study cases for the applicability of the presented work is acknowledged. The Technical University of Demark (DTU), Kgs. Lyngby, Denmark is acknowledged for funding the PhD program of the corresponding author. Furthermore, acknowledgement is provided to Engel Denmark A/S, Solrød Strand, Denmark, (subsidiary of Engel Austria $\mathrm{GmbH}$, Schwertberg, Austria) which provided technical data about the used injection molding machine for setting up the simulations.

\section{References}

[1] Vietri U et al. Polymer Engineering and Science. 51:12 (2011) 2542-2551.

[2] Tosello G et al. Proceedings of Polymer Process Engineering 11 - Enhanced Polymer Processing (2011) 413-435.

[3] Ruprecht R et al. Microsystem Technologies. 8:4-5 (2002) 351-358.

[4] Kazmer DO. Injection Mold Design Engineering, Hanser, 2007.

[5] Kietzmann $C$ et al. Proceedings of the $72^{\text {nd }}$ Annual Technical Conference ANTEC (2013), 6 pages.

[6] Zhiltsova TV et al. Journal of Material Science. 48:1 (2013) 81-94. 